\title{
Et si la climatologie devenait une science sociale comme les autres ? À propos du colloque «Entre connaissance et action: regards croisés sur les enjeux climatiques et environnementaux »
}

\author{
Francis Chateauraynaud $^{1, *}$ et Cathy Dubois ${ }^{2, a}$ \\ 1 Sociologie, EHESS, Directeur du Groupe de sociologie pragmatique et réflexive, Paris, France \\ 2 Sociologie, R\&D consultants, Paris, France
}

\begin{abstract}
Résumé - Cet article revient sur les échanges qui ont eu lieu lors du colloque «Entre connaissance et action: regards croisés sur les enjeux climatiques et environnementaux » organisé au Centre national d'études spatiales à Paris en décembre 2016. Les climatologues évoquent parfois une certaine indifférence des sciences humaines et sociales face à leurs appels à la mobilisation pour « la cause du climat». Or, des programmes de recherche récents ont favorisé des coopérations entre chercheurs de différentes disciplines, révélant aux participants l'intérêt de confrontations continues de leurs approches. Cette synthèse critique restitue les principaux enseignements de cette journée et invite à aller encore plus loin, en considérant la climatologie comme une science sociale capable de relier logique axiomatique et logique phénoménologique.
\end{abstract}

Mots-clés : enjeux climatiques et environnementaux / interdisciplinarité / recherche intégrée / formes de vie / multi-échelles / citoyenneté distribuée

\begin{abstract}
Might climatology become a social science like others? On the conference "Between knowledge and action: cross perspectives on climate and environmental issues". This article reconsiders the debates which took place during the conference "Between knowledge and action: cross perspectives on climate and environmental issues" held in December 2016 at the Centre national d'études spatiales headquarters in Paris. Climatologists sometimes mention feeling a certain indifference of the human and social sciences vis-à-vis their calls for mobilization in defense of the "climate cause". Recent research programs have stimulated cooperation between researchers from various disciplines thus making participants aware of the interest of a continuous confrontation between their different approaches. This critical synthesis reports on the main lessons of the meeting and invites to move forward by considering climatology as a formal social science that could link axiomatic and metrological reasoning on data with phenomenology and social investigations "on the ground".
\end{abstract}

Keywords: climatic and environmental stakes / interdisciplinarity / integrated research / forms of life / multi-scales / distributed citizenship

À l'initiative du Groupement d'intérêt scientifique Climat-Environnement-Société (GIS Climat), du programme du Centre national d'études spatiales (CNES) «Espace, innovation et société », et en collaboration avec le programme Gestion et impacts du changement climatique (GICC) du ministère de l'Environnement, de

\footnotetext{
*Auteur correspondant : chateau@msh-paris.fr

${ }^{a}$ C. Dubois a été la coordinatrice du programme de recherche Espace-innovation-société du Centre national d'études spatiales (2012-2016).
}

l'Énergie et de la Mer, la conférence «Entre connaissance et action: regards croisés sur les enjeux climatiques et environnementaux » était organisée le 15 décembre 2016 à Paris. Destinée à croiser les expériences, elle fut l'occasion d'amorcer une réflexion prospective autour des recherches produites par les différents programmes impliqués. L'expression et la confrontation des positionnements d'équipes françaises face aux évolutions des problématiques environnementales à l'échelle internationale, ont ainsi permis d'entrevoir les enjeux de recherches futures mobilisant sciences du climat et sciences sociales. 
Depuis la Conférence de Paris de 2015 sur les changements climatiques (COP21), la matrice discursive du changement climatique a renforcé la logique de l'urgence face à la catastrophe à venir, urgence motivant des injonctions officielles qui insistent désormais sur l'engagement de processus d'adaptation. Cela faisait plusieurs années que l'adaptation avait rejoint les appels à la réduction des émissions de gaz à effets de serre (GES), le scénario d'un «retour à la normale» au cours $\mathrm{du} \mathrm{XXI}^{\mathrm{e}}$ siècle étant jugé de plus en plus improbable. Tous les participants au colloque en sont convenus : le passage de la problématique de la réduction des émissions, qui engage déjà des sciences sociales via les enjeux économiques, politiques et juridiques du «gouvernement mondial du climat» (Aykut et Dahan, 2015), à celle de l'adaptation, implique un changement d'échelle (Treyer et Aubertin, 2016) ou plutôt la prise en compte d'interactions complexes entre différentes échelles de représentation et d'action. Il ne suffit pas de zoomer/dézoomer sur des localités. Il faut se placer au plus près des acteurs aux prises avec les manifestations et les perspectives locales ou régionales du réchauffement planétaire, tout en prenant en compte les multiples boucles de rétroaction liant des dynamiques sociales, forcément situées, et des dispositifs normatifs, éminemment globalisés. L'accord de Paris, dont nombre de commentateurs ont annoncé la fragilité constitutive sous l'apparente solidarité générale, énonçait la nécessité de lier plusieurs échelles d'analyse et d'action. Lors du colloque de décembre 2016, plusieurs chercheurs ont évoqué la nécessité d'un changement de paradigme, accordant plus d'importance aux initiatives locales des acteurs, afin de privilégier des recherches intégrées liant toujours plus étroitement production de connaissances et appui à l'action. Il est apparu que d'autres modalités de coopération restaient à inventer au cœur des interactions entre les multiples champs scientifiques convoqués par le climat et la biodiversité. En invitant dans le titre de cet article à considérer la climatologie comme une science sociale, il s'agit de surmonter les vieilles fractures entre trois manières de dire le monde: les démarches axiomatiques - ce dont relève la modélisation du « système Terre »-, les approches constructivistes - qui mettent l'accent sur la fabrique des règles et des conventions communes - et l'entrée par les expériences situées au cœur des innombrables versions du monde matériel et sensible coproduites par les acteurs dans leurs cours d'action ordinaires.

\section{Trois régimes de preuve qui font la tangibilité du changement climatique}

L'étude approfondie de nombreuses controverses a conduit à identifier trois régimes de preuve (Chateau- raynaud et Debaz, 2017), nécessaires à la bonne interprétation des processus à l'œuvre. Le premier régime de preuve est qualifié d' "axiomatique», puisqu'il dépend de la production d'un système formel, et se déduit d'un espace de calcul, en vertu de ce qu'on peut appeler une axiomatique autonome. Une fois rendue autonome, une axiomatique peut être automatisée. Les exemples peuvent être pris dans bien des domaines, mais, du côté des sciences, l'astrophysique fait figure d'avant-garde avec une insolente capacité de prédiction portant sur des univers inaccessibles aux sens, du moins sans de longues chaînes de médiations instrumentales. Dans une axiomatique autonome, les règles ne peuvent pas être changées librement par les acteurs et les modes de représentation deviennent euxmêmes accessoires - l'objet mathématique étant souvent iconoclaste. Quelle est la part d'axiomatisation, ou si l'on préfère de formalisation, dans la conception et la mise en œuvre des instruments de la climatologie ? C'est une des dimensions à examiner en priorité à la fois pour discuter des domaines de validité et pour envisager la production d'agencements ou d'articulations de méthodes hétérogènes, dont la convergence peut faire naître une nouvelle axiomatique.

Le deuxième régime, appelé régime conventionnaliste, a beaucoup occupé l'histoire des sciences, suscitant d'intenses controverses autour du relativisme ou du constructivisme. Dans ce régime, pour fabriquer une preuve, il faut tomber d'accord sur des ontologies, c'est-àdire sur des catégories et des règles d'équivalence. C'est pour cette raison que l'on peut y voir le règne des constructions sociales (Daston et Galison, 2007). Pour fournir des preuves statistiques, par exemple, on doit construire des ensembles (des populations) et des descripteurs (des variables), autant d'outils de caractérisation qui n'échappent qu'exceptionnellement au flou des catégories et de leurs frontières toujours liées à un travail sémantique, lui-même dépendant de luttes définitionnelles, dans lesquelles sciences et politiques interagissent intensément. Des preuves statistiques sont possibles, à condition de stabiliser les catégories, lesquelles supposent des conventions solides. Ce sont ces conventions que prétendent dépasser ou relativiser les démarches rassemblées sous l'appellation de big data, et qui réveillent les tensions entre régime axiomatique (syntaxique ou formel) et régime conventionnaliste (sémantique ou ontologique).

Mais on ne peut en rester à cette opposition, car un troisième régime est à l'œuvre: un régime phénoménologique de la preuve. C'est ce régime que pratiquent spontanément les sciences sociales lorsqu'elles prennent appui sur les expériences du monde et sur les formes d'intercompréhension, les prises communes, qu'elles rendent possibles. On parlera aussi bien de régime pragmatique ou écologique de la preuve. C'est là un point 
important pour la suite: les relations entre ce régime phénoménologique et les deux premiers passent par des opérations interprétatives dont la fonction est de surmonter, par et pour des collectifs d'acteurs, les incomplétudes (celles des systèmes formels), les incertitudes (celles des conventions sur les états du monde) et les irréductibilités (celles des expériences subjectives et des contextes dans lesquels celles-ci prennent forme).

Le devenir des interactions entre approches formelles du climat et approches conventionnalistes et phénoménologiques des dispositifs et des milieux les plus divers, réside dans l'organisation des relations entre les trois régimes en permettant une circulation et une réflexivité permanente à toutes les échelles de représentation et d'action.

\section{Penser les contre-anthropocènes à partir des interactions entre dispositifs et formes de vie}

Cette approche plurielle des régimes de connaissance permet de penser les dynamiques locales en sortant de la seule logique adaptative conçue du point de vue global. Elle donne à voir les multiples contreanthropocènes engendrés par les milieux en interaction (Chateauraynaud et Debaz, 2017). L'idée de contreanthropocène désigne les milieux résistants et résilients qu'invoquent les acteurs, le plus souvent à l'appui de contestations ou de protestations. Mais elle vise aussi, et surtout, tout assemblage polyphonique, au sens que donne à cette formule Anna Tsing dans son ouvrage consacré à la multiplicité des formes de vie qui naissent dans les restes, les interstices, les ruines du capitalisme (Tsing, 2015). La question des échelles temporelles et spatiales d'appréhension des phénomènes, comme celle de leurs angles d'analyse, joue ici un rôle déterminant. Toutefois l'articulation des approches reste délicate sans une pragmatique de la complexité capable de saisir des dynamiques non linéaires (Chateauraynaud, 2016). Les travaux en climatologie mettent déjà en avant une forme de complexité mais ils portent sur un macro-monde, mobilisent des modélisations numériques, et visent les principaux points et facteurs de rupture des grands équilibres climatiques. Les travaux en sciences humaines et sociales (SHS) s'intéressent à des acteurs situés, même lorsqu'ils œuvrent au nom d'entités inter- ou transnationales (Charvolin et Ollivier, 2017), privilégiant des études ancrées dans des réalités géographiques dont l'articulation aux problématiques planétaires globales, relève d'espaces de mobilisation et de jeux institutionnels complexes. La transformation des grands équilibres, provoquée par différentes manières d'habiter la Terre, affecte l'humanité mais pas de manière homogène. Une grande asymétrie de situations nourrit déjà le développement de tensions, de guerres et des migrations climatiques.

La question climatique est donc d'emblée tout à la fois physique et politique. Inviter aujourd'hui à la prendre comme une question de sciences sociales, c'est proposer de s'intéresser autant aux capacités de reconfiguration qui émergent au sein des sociétés humaines contemporaines (Tsing, 2015) qu'aux dynamiques qui les conduisent à dégrader leurs milieux jusqu'à les rendre inhabitables. Ces reconfigurations peuvent être multiples, technologiques et/ou sociales, chercher à réparer le monde physique (géo-ingénierie), à modifier les manières de l'habiter (décroissance) ou à transformer les êtres humains (transhumanisme); toutes mobilisent des visions du futur plus ou moins conflictuelles, se déploient sur des trajectoires de changements plus ou moins contradictoires, privilégiant certains acteurs plutôt que d'autres. Dans cette perspective, élaborer des connaissances sur les transformations en cours est en soi un enjeu politique. La définition des objets d'analyse, des instruments et des calendriers de mesure, l'usage de ces mesures, les choix opérés pour les politiques scientifiques elles-mêmes refléteront la capacité des sociétés à s'emparer réellement du problème.

\section{Du global au local : déplacer les regards sur les jeux d'échelle}

De 2007 à 2016, le GIS Climat-EnvironnementSociété fondé à l'initiative des climatologues ${ }^{1}$, a eu pour mission de développer la recherche interdisciplinaire sur le changement climatique et ses impacts, à partir d'un consortium regroupant dix-sept laboratoires, travaillant dans les domaines du climat, de l'économie, de l'écologie, de l'hydrologie, de la santé et des sciences humaines et sociales. Le projet a été initié par l'Institut Pierre-Simon-Laplace, dans le but d'étendre la démarche d'ouverture interdisciplinaire déjà entreprise, tout en conservant la perspective d'une approche «système Terre ». Au niveau international, des approches similaires à celle du GIS Climat ont été développées dans le cadre du partenariat Earth System Science Partnership. Pendant la même période, le programme de recherches interdisciplinaire du CNES «Espace, innovation et société », a favorisé des coopérations de longue durée

\footnotetext{
${ }^{1}$ Sous la direction de Sylvie Joussaume (CNRS) et Chantal Pacteau (CNRS), le Groupement d'intérêt scientifique (GIS) Climat-Environnement-Société a réuni 17 laboratoires d'Îlede-France travaillant principalement dans les domaines de la climatologie, l'hydrologie, l'écologie, la santé et les sciences humaines et sociales : http://www.gisclimat.fr/
} 
entre des ingénieurs du CNES et des chercheurs de différents laboratoires de SHS $^{2}$.

Saisis comme points d'observation surplombants, affectés d'une supposée neutralité territoriale et d'une objectivité métrologique rarement contestée, les satellites sont parfois considérés comme des instruments privilégiés pour l'évaluation des contributions des États à la lutte contre le changement climatique. La réalité est plus complexe. Les chercheurs du programme ont ainsi mis en évidence qu'une contribution effective des dispositifs spatiaux aux grands défis de la société requérait d'élargir le cercle des acteurs engagés dans la discussion des problématiques et des mesures, de l'aval, via les usages, jusqu'à l'amont, au cœur de la conception des dispositifs spatiaux. La réflexion sur les usages possibles des données produites grâce aux dispositifs satellitaires pour la gouvernance mondiale du climat, la gestion du carbone et les politiques de développement, peut favoriser la révision du mode de coopération entre ingénieurs, scientifiques et autres acteurs sociaux. Les scientifiques et les ingénieurs sont en effet souvent interpellés par des problématiques élaborées par d'autres acteurs.

Depuis sa construction comme question scientifique et politique par les climatologues, le changement climatique est un objet complexe à plus d'un titre. Le Giec (Groupe d'experts intergouvernemental sur l'évolution du climat) et les communautés scientifiques qui l'ont constitué ont élaboré des formules comme la «température moyenne» de la Terre ou la «montée du niveau des océans »; la problématique principale qu'ils ont développée est centrée sur les transformations du régime climatique du système Terre. Les indicateurs les plus couramment utilisés, faussement simples, sont issus d'un long processus d'élaboration de l'espace de calcul climatique, fondé sur un large éventail de mesures et de simulations (Edwards, 2010; Aykut et Dahan, 2015). Forgés pour l'étude des tendances longues et adaptés à la modélisation numérique du régime climatique, les instruments procurent des appuis métrologiques pour l'observation, l'alerte et la mobilisation politique-au sens de «policy relevant» maintes fois souligné par le

\footnotetext{
${ }^{2}$ Les sociologues du Groupe de sociologie pragmatique et réflexive de l'École des hautes études en sciences sociales (EHESS) et du laboratoire Printemps (CNRS, Université de Versailles-Saint-Quentin-en-Yvelines); des historiens du Centre Alexandre-Koyré (CNRS, EHESS, MNHN), des chercheurs en gestion du Centre de recherche en gestion de l'École polytechnique ainsi que du Centre de gestion scientifique de l'École des mines (fusionnés dans le laboratoire I3 [CNRS, École polytechnique, Mines ParisTech, Télécom ParisTech]), les géographes du laboratoire Passage de Bordeaux (CNRS, Université de Bordeaux, Université Bordeaux-Montaigne, Université de Pau et des pays de l'Adour, ENSAP Bordeaux).
}

Giec. Mais au-delà de leur dimension instrumentale, ces opérations ont contribué à faire émerger le climat comme un bien commun de l'humanité. Les acteurs sociaux ont souvent du mal avec ce concept d' «humanité» dont l'abstraction et la globalisation masquent les asymétries de situation (Hulme, 2013).

La prise en compte des expériences des acteurs suppose de dépasser la seule figure d'un «nous totalisant» et de mettre à juste distance les annonces catastrophistes sur la montée inéluctable des risques et des menaces globales (Chateauraynaud et Debaz, 2017). Les textes de présentation du One Planet Summit organisé à Paris par le Président Emmanuel Macron en décembre $2017^{3}$, deux ans après l'accord de Paris, montrent à la fois la progression et la difficulté d'un tel changement de perspective. $\mathrm{Si}$, dans une posture réaliste, les enjeux d'atténuation et d'adaptation sont intimement liés, le «nous» d'une humanité globale et solidaire domine le discours: "Nous sommes une seule planète». L'injonction à l'action, qui se fonde sur l'urgence, prend la forme d'un appel à l'innovation et à la mobilisation de tous dans la mise en œuvre d'actions concrètes aux différentes échelles, globales et locales. On soupçonne une hiérarchisation encore très classique des échelles d'action mais il est malgré tout question de concevoir des politiques publiques soutenant l'action des gouvernements locaux et régionaux. Dans le même mouvement, la finance publique reçoit pour mission de faire levier sur l'investissement privé.

\section{Mettre au cœur des recherches les relations entre adaptation au changement climatique et formes de vie}

La notion de système Terre, au cœur de l'étude physique du climat, ne renvoie pas à l'expérience sensible des acteurs, ni à leur capacité d'action-elle a même été construite en rupture avec le «sens commun». Pour les sociologues, les anthropologues ou les politistes, les formulations en termes de «système humain» ou «facteur humain», utilisées parfois par des climatologues pour pointer les effets des activités humaines sur l'environnement et les grands équilibres climatiques, sont trop globales pour permettre l'analyse des dynamiques sociales, parce que trop éloignées des expériences sensibles des acteurs sociaux et des interprétations qu'ils leur donnent (Ingold, 2011; Cometti, 2015 ; Crate et Nuttall, 2016). Les sciences de l'homme et de la société s'intéressent plutôt aux relations que les acteurs entretiennent avec leurs milieux de vie, aux interactions complexes et aux frictions entre éléments géophysiques et sociétés, ou au façonnage des paysages par les sociétés

\footnotetext{
$\overline{{ }^{3} \text { https://www.oneplanetsummit.fr/fr/ }}$
} 
humaines, avec tous les enjeux qui les accompagnent (Blanc et al., 2017).

Les formes de vie, les façons d'habiter le monde et d'élaborer des médiations pratiques entre les milieux en interaction sont au cœur des observatoires de la biodiversité. Mais elles ne sont pas encore bien prises en compte dans la construction des problématiques climatiques (Borie et Hulme, 2015). Si la trajectoire ascendante de la cause climatique repose avant tout sur l'espace de calcul des émissions et des courbes de température moyenne, et sur l'idée, chère à de nombreux global thinkers, selon laquelle seule une pensée globale permet de prendre conscience des enjeux, jugés imperceptibles aux échelles locales, il est maintenant nécessaire de penser, dès l'amont, des dispositifs de recherche et d'action plus symétriques. Une des clauses du cahier des charges est pour le moins d'aller au-delà de la seule reconnaissance des savoirs locaux comme adjuvants, comme facilitateurs d'acquisition de données ou d'ajustements des paramètres métrologiques. Ce que les promoteurs de standards d'évaluation et d'action publique appellent «descente en échelle» fait (presque toujours) surgir des divergences entre les acteurs tant pour l'évaluation des problèmes, la qualification des situations, que du point de vue des visions du futur. Un des enjeux est ainsi de penser à la fois le climat comme bien commun mondial, appuyé sur la modélisation du système Terre et de fédérer, sans les réduire, des manières très différentes de construire des communs à d'autres échelles, fondées parfois sur des ontologies ou des cosmologies incommensurables. Autrement dit, il est temps de donner corps à la figure de la gouvernance polycentrique défendue par Elinor Ostrom ${ }^{4}$.

\section{Inventer des pratiques interdisciplinaires aussi indisciplinées que possible}

Une analyse des travaux interdisciplinaires menés par le GIS Climat a été effectuée à partir d'entretiens avec les porteurs de projet (Joussaume et al., 2016). Elle a permis de mettre en lumière trois types d'approches, qualifiées ici dans les termes des chercheurs eux-mêmes: une fertilisation croisée, une production hybridée et l'émergence d'approches intégrées. L'observation des différents travaux a par ailleurs conduit à inventer et à tester de nouvelles approches réflexives. Du côté du CNES, les chercheurs ont réfléchi aux conditions d'une contribution effective des dispositifs spatiaux aux grands défis

\footnotetext{
${ }^{4}$ Voir le dossier «Le champ des commons en question: perspectives croisées », Natures Sciences Sociétés, 19-20, 4-1, 2011-2012.
}

sociétaux. Les échanges avec les sciences sociales ont conduit à réviser la forme de dialogue, historiquement constituée, entre ingénieurs, scientifiques et autres acteurs sociaux. Classiquement, il revient aux scientifiques de définir les mesures à effectuer et de produire des connaissances à partir de ces mesures, aux ingénieurs de concevoir les instruments et les missions, aux autres acteurs sociaux de traduire ces connaissances et d'investir les outils pour des usages sociaux précis. Élargir le cercle des acteurs engagés dans la construction des problématiques et la conception des dispositifs spatiaux du futur paraît d'autant plus indispensable que l'émergence de nouvelles technologies et de nouveaux acteurs bouleversent les modes traditionnels de financement de ces dispositifs.

En se fondant sur l'expérience des trois programmes cités plus haut, le comité scientifique de la conférence de décembre $2016^{5}$ a convié des chercheurs d'horizons différents (Encadré 1) à revenir sur leurs enquêtes et leurs coopérations, afin de réfléchir aux conditions de fertilité de recherches interdisciplinaires intégrées, dans une double perspective de production de connaissances et de réponse aux enjeux sociétaux. La ville, l'échelle régionale, les risques côtiers, les services climatiques étaient les quatre entrées thématiques retenues. Elles renvoient assez directement au registre de l'adaptation, tout en recouvrant des réalités sociales différentes (en termes d'organisation sociale, de niveaux administratifs, de détermination des risques ou de dispositifs informationnels visant des usagers spécifiques). A posteriori, ces quatre entrées illustrent ce que pourrait signifier «travailler la climatologie comme une science sociale $»$ : le cadrage et la construction des problématiques, tout autant que la prise en compte ou non des initiatives émergentes, transforment la production des connaissances et par là même la possibilité pour de nouvelles communautés d'acteurs de se saisir de ces connaissances pour l'action.

\section{La ville : un territoire à échelle inhumaine?}

La ville, modèle de vie prédominant ${ }^{6}$, offre l'opportunité d'aborder deux enjeux de la coopération entre

\footnotetext{
${ }^{5}$ Michel Avignon (CNES), Gilles Bergametti (CNRS), Jean-Paul Billaud (CNRS), Francis Chateauraynaud (EHESS), Cathy Clerbaux (CNRS), Cathy Dubois (R\&D Consultants), Maurice Imbard (MTES), Sylvie Joussaume (CNRS), Patrick Monfray (MESRI), Chantal Pacteau (CNRS), Jean-Paul Vanderlinden (UVSQ), Denis Salles (Irstea).

${ }^{6}$ En 2017, 53\% de la population mondiale vivait en zone urbaine. Plusieurs scénarios annoncent $67 \%$ pour 2050 . En France, $75 \%$ de la population française est en zone urbaine ou péri-urbaine.
} 
climatologie et SHS dans la perspective de l'appui aux politiques d'adaptation: le rôle de la végétalisation et la qualité de l'air. La végétalisation des espaces urbains, dont on connaît surtout l'exemple des trames vertes, est considérée comme une ressource pour l'adaptation des villes au changement climatique. En analysant les productions scientifiques sur le sujet, le sociologue Philippe Boudes a montré la coexistence de trois formes de relation entre végétation et climat en ville: la végétation comme réalité scientifique, comme processus socioculturel et comme variable des politiques d'aménagement. Dans la littérature - celle du Giec ou celle du Grenelle de l'environnement-, le registre scientifique et le registre politique dominent. Le registre phénoménologique, celui de l'expérience sensible et cognitive des individus et des groupes de leur appropriation des enjeux de nature, est moins présent, alors même que les politiques de végétalisation territorialisent la question du climat à l'échelle du cadre de vie des personnes. Engagé dans le même projet de recherche pluridisciplinaire «Changement climatique et trames vertes» $\left(\mathrm{CCTV}^{7}\right)$, le politiste Aleksandar Rankovic a fait remarquer qu'il n'existe pas une conception monolithique de la végétation: s'il est traité comme «réfrigérateur» dans la littérature au détriment d'une prise en compte de sa dynamique propre, le végétal urbain est saisi dans des imaginaires d'une nature idéalisée mais aussi dans les représentations sociales associées aux métiers qui interviennent dans sa gestion. De plus, l'arbre en ville est inscrit dans un contexte plus large qui inclut aussi bien les jardins partagés que la gestion des trottoirs.

En abordant les interactions entre climat, qualité de l'air, urbanisme et santé publique, Isabelle Coll a insisté sur les enjeux d'une approche intégrée tant pour la production de connaissances que pour l'action. La dynamique des zones urbaines est peu favorable à la santé. Ce sont des zones qui consomment beaucoup de ressources et exercent des pressions importantes sur l'environnement. Par leur densité de population, leurs activités économiques et le volume des déplacements, les villes sont des sources majeures de pollution atmosphérique. Du même coup, les enjeux de transformation y sont considérables, tant du point de vue de l'atténuation des émissions que de l'adaptation aux changements climatiques. Les villes deviennent, de fait, de véritables laboratoires d'autres manières d'habiter dont les ressorts ne peuvent se limiter à des dimensions technologiques. Les campagnes de mesures, l'établissement de corrélation avec des problématiques épidémiologiques, la production de données scientifiques, ne

\footnotetext{
${ }^{7}$ CCTV 1 et 2, programmes de recherche du GIS Climat en 2009-2010, puis 2011-2014 avec l'appui de l'Ademe.
}

Encadré 1. Liste des interventions citées dans le texte.

Réflexions sur les «nouveaux paradigmes» et les enjeux futurs de coopération

- Entre anticipations et ruptures, l'émergence des futurs. Logiques computationnelles, expériences des milieux et ouvertures d'avenir. Francis Chateauraynaud (GSPR-EHESS)

- Enjeux climatiques : vers une démarche plus intégrée avec les sciences humaines et sociales, l'expérience $\mathrm{du}$ GIS Climat. Sylvie Joussaume (CNRS-GIS Climat)

Problématiques à l'échelle de la ville

- Trames vertes urbaines: une option d'adaptation face au changement climatique? (projet CCTVGIS Climat). Chantal Pacteau (CNRS-GIS Climat), Aleksandar Rankovic (IEES-IDDRI) et Philippe Boudes (Agrocampus Ouest \& ESO CNRS)

- Climat, qualité de l'air et santé publique en milieu urbain: les enjeux d'une prise en compte intégrée. Isabelle Coll (Université Paris-Est-Créteil-Lisa)

- La pollution de l'air et les lobbies face aux défis climatiques: reproduction des mêmes erreurs? Chloé Vlassopoulos (Université de Picardie-JulesVerne)

Préparer/Accompagner à l'échelle d'un territoire régional

- Science, société, politique. Les leçons d'Acclimaterra, observatoire régional du changement climatique en Nouvelle-Aquitaine. Hervé Le Treut (CNRS-IPSL) et Denis Salles (Irstea)

- Changements climatiques en Afrique subsaharienne : vulnérabilité et adaptation des sociétés rurales (projet ESCAPE-GIS Climat). Benjamin Sultan (IRDLocean-UPMC) et Bénédicte Gastineau (IRD-Aix Marseille Université)

Appréhender les risques côtiers

- Changements environnementaux côtiers. Carlos Oliveros (BRGM)

- Cultures et mesures du risque : clivages et coalitions en milieu littoral. Josquin Debaz (GSPR-EHESS) et Arnaud Saint-Martin (CNRS)

De la conception des «services» à l'action opérationnelle

- Services climatiques: Histoire d'une métamorphose? Ou comment passer du statut de victime à celui d'acteur. Pascale Braconnot (IPSL-CEA), Florence Rudolf (INSA-Strasbourg) et Amandine Amat (INSA-Strasbourg)

- La construction de la valeur des données spatiales. Sylvain Lenfle (CNAM-I3) suffisent pas à amorcer des politiques vertueuses. Les expérimentations démocratiques jouent un rôle clé, notamment lorsque les citadins sont invités à passer 
d'une économie de propriété à une économie d'usage collectif, d'une posture "d'usager d'une ville pensée pour eux» à une posture de «coproducteur d'une ville en transition ». Pour lever les verrous du changement, I. Coll a appelé à mettre en place une transdisciplinarité dans l'aide à la décision et l'évaluation des politiques publiques, en croisant l'urbanisme, l'architecture, l'agriculture, les sciences de l'environnement, la sociologie, l'économie, l'épidémiologie, les statistiques et la toxicologie. La coproduction et le partage des données concernant la santé, l'environnement ou les statistiques d'urbanisme sont des leviers pour des reconfigurations plus profondes que les seuls aménagements de surface.

De son côté, Chloé Vlassopoulos a rappelé que la connaissance scientifique et la publicisation des données ne suffisent pas à entraîner une mise en place effective de politique publique. L'exemple des véhicules diesel en France est patent : la nocivité des émissions de particules était déjà notifiée dans des rapports publics du début du $\mathrm{XX}^{\mathrm{e}}$ siècle. La part des véhicules diesel dans le parc automobile français est néanmoins passée de $8,15 \%$ en 1980 à $68 \%$ en 2015. On sait que la Commission européenne a pris le parti du véhicule électrique, considérant la question du climat comme une opportunité pour le développement de cette technologie. Mais les réseaux électriques peuvent-ils suivre? L'industrie automobile française est longtemps restée prise entre la défense du statu quo, au motif que les moteurs diesel émettraient moins de $\mathrm{CO}_{2}$ que les moteurs à essence, et la promotion d'une technologie alternative offrant de nouveaux horizons économiques - mais dont le caractère proprement «écologique» est sujet à caution. Dans les approches de l'adaptation, les groupes et réseaux d'influence cherchent à anticiper et configurer la rédaction des normes régulatrices selon qu'elles sont favorables ou défavorables à leur activité. En tout état de cause, comme indiqué plus haut, il serait naïf de considérer que les divergences d'intérêt peuvent facilement s'effacer dans un "nous», forme pronominale d'une humanité une et indivisible, subitement solidaire face aux enjeux climatiques.

\section{L'échelle régionale : accroître les capacités des acteurs à éprouver des situations inédites}

La mise en relation de travaux menés en Aquitaine d'une part, et en Afrique subsaharienne d'autre part, a permis d'aborder la question de l'apport de la recherche à l'action publique, à travers des acteurs régionaux ou locaux: appui à la compréhension et à l'appropriation des problématiques locales du changement climatique, aide à la scénarisation du futur et à la décision. En Aquitaine, la région a fait appel à des scientifiques, regroupés dans un comité dénommé Acclimaterra $^{8}$, pour comprendre en quoi le territoire est climato-dépendant. À partir du rapport collectif qu'il a coordonné, Hervé Le Treut a expliqué les enjeux d'une articulation des problématiques climatiques globales et locales, en montrant les incidences à l'échelle locale des changements globaux en cours, ce qui incite à informer et sensibiliser les Aquitains (acteurs économiques, associations, grand public) aux adaptations nécessaires (Le Treut, 2013). Après un premier rapport, puis un livre présentant les résultats en fonction de grandes thématiques d'application, les chercheurs d'Acclimaterra ont compris que répondre à des questions que les gens ne se posent pas n'est guère suffisant pour susciter l'action. Ils ont donc adopté une perspective inversée en se fondant sur des questions exprimées par les habitants lors de réunions publiques, organisées avec l'aide du sociologue Denis Salles, dans les six grandes villes de la région. Ils ont également choisi de donner une place différente aux ONG. Ces rencontres qui ont permis de faire remonter les interrogations des associations obligent à un retour en fin de processus. De ce processus, les climatologues retiennent que, malgré leurs efforts, la connaissance et la prise de conscience par les citoyens et les élus $\mathrm{du}$ problème $\mathrm{du}$ changement climatique sont loin d'être à la hauteur des enjeux. Les difficultés majeures de communication publique portent sur les notions d'aléa et d'incertitude, car si les scientifiques savent bien expliquer ce qui peut se produire à très grande échelle, il leur est plus difficile de se prononcer sur des processus locaux. De plus, comme pour la ville, en l'absence de bases de données intégrées, il est difficile de produire une vision synthétique, d'obtenir une carte d'identité environnementale d'une région, carte qui doit être nécessairement évolutive. L'approche de l'adaptation gagnerait alors à être reformulée en termes d'articulation entre politiques publiques, perceptions des populations et vulnérabilité des milieux aux changements climatiques et environnementaux.

La question est particulièrement sensible en Afrique où de nombreuses zones sont considérées comme très vulnérables. Mais, loin de tout catastrophisme, l'enquête permet d'observer de multiples stratégies d'adaptation, en réaction aux événements extérieurs. Bénédicte Gastineau et Benjamin Sultan, chercheurs du programme

\footnotetext{
${ }^{8}$ Acclimaterra est un comité scientifique, né du souhait du conseil régional de Nouvelle-Aquitaine, qui rassemble des experts capables d'apporter aux acteurs du territoire les connaissances nécessaires à leur stratégie d'adaptation au changement climatique.
} 
pluridisciplinaire $\operatorname{ESCAPE}^{9}$ (associant spécialistes du climat, océanographes, anthropologues, historiens, démographes et agronomes), ont étudié les capacités de sociétés de l'Afrique subsaharienne à transformer leur fonctionnement selon leurs vulnérabilités. Les climatologues ont dressé le bilan des grands changements environnementaux accumulés au cours des dernières années pour les confronter aux transformations sociales et économiques dans les régions concernées, et identifier éventuellement des liens de causalité. Les chercheurs ont tenté de comprendre comment, à l'échelle locale, les paysans et les sociétés rurales avaient perçu ce type de changement. L'enquête montre que les évolutions sociales doivent être appréhendées de manière globale et sur le long terme, surtout lorsque les acteurs locaux n'établissent pas de liens directs avec le changement climatique. Les nouvelles pratiques agricoles sont corrélées à la volatilité des prix, à des choix alimentaires et à des problématiques politiques locales.

Les pratiques d'adaptation (démographie, migration, transformation des pratiques agricoles ou des règles de transmission des terres...) puisent dans des références à des cycles, à des événements passés ou aux pratiques des anciens. La question de la manière dont les sociétés s'adapteront aux situations engendrées par des changements environnementaux en cache une autre: celle que soulèvent les projections qui portent à penser que le climat de demain ne ressemblera pas au climat d'aujourd'hui. Les chercheurs s'interrogent sur les limites de la plasticité des sociétés et sur leurs ressources réelles de transformation en situation inédite. Comment les chercheurs peuvent-ils coconstruire avec les acteurs une adaptation planifiée dans le futur, orienter vers de nouvelles variétés et de nouvelles techniques pour lutter contre les effets du changement climatique?

\section{Les zones côtières : la fluctuation des périmètres de solidarité}

La question des zones côtières, autre thématique majeure du colloque, introduit une dimension supplémentaire puisque la sensibilité et la vulnérabilité du milieu à l'aléa configurent directement ce qu'il est convenu d'appeler le «territoire du risque». Or, loin d'être une donnée figée, la vulnérabilité est inscrite dans des processus sociaux: pressions urbaines, type d'activités économiques, tourisme, infrastructures de transport... Il est dès lors étonnant de voir perdurer des approches top-down et linéaires considérant l'adaptation

\footnotetext{
${ }^{9}$ Le projet ANR ESCAPE (Études rétrospectives et prospectives sur l'évolution de différents systèmes sociaux, agricoles et écologiques dans le contexte des changements environnementaux, 2011-2015) comptait 8 partenaires français et 10 partenaires africains (Sénégal, Mali, Niger et Bénin).
}

comme une réponse à un aléa «naturel». D'autant plus que si la montée du niveau des mers est une certitude, les évolutions du littoral, que ce soit dans le temps court de l'action ou à plus long terme, sont difficiles à prévoir. Les reconfigurations s'y opèrent sous l'influence de plusieurs dynamiques en interaction. Quant à la relation directe entre le changement climatique et les risques d'érosion ou de submersion, elle n'apparaît évidente, comme l'ont montré les études du projet CECILE ${ }^{10}$, que dans les zones arctiques et pour les petites îles. Dans le cas des îles du Pacifique, des événements climatiques comme les cyclones produisent tantôt de l'érosion, tantôt de l'accrétion sur certaines parties du littoral.

La complexité des trajectoires suivies par les zones littorales fournit un motif supplémentaire pour le lancement de recherches intégrées réunissant des chercheurs de plusieurs disciplines et notamment des SHS. Les sociologues Josquin Debaz et Arnaud SaintMartin ${ }^{11}$ ont témoigné de leurs difficultés à faire financer des projets allant dans ce sens. Est-ce parce qu'ils insistent sur la dimension politique de la vulnérabilité ? À considérer le risque comme un objet technique ou à travers les seuls filtres de l'administration de la prévention, on perd de vue que la catastrophe est toujours «un débordement» des cadres connus, une rupture avec ce qui avait pu être anticipé. Et en situation, les acteurs ne disposent pas tous des mêmes ressources: expériences d'événements similaires, mémoire collective, dispositifs de secours, accès à des alternatives, possibilité ou non de déployer des stratégies de protection... L'intérêt des sciences humaines et sociales est d'introduire de la réflexivité et de la critique en ne taisant ni les frictions ni les conflits. D'où l'enjeu de comprendre comment les choses fonctionnent «par le bas» et de ne pas fermer les yeux quand les « cultures du risque » font défaut ou contribuent - le plus souvent de façon non intentionnelle - aux dysfonctionnements. Comment les politiques du risque sont-elles influencées? Comment les acteurs et les institutions prennent-ils en compte les attentes à l'égard de la métrologie et des modèles? Approcher les risques côtiers, du côté des populations, pas seulement après le désastre, c'est entrer dans l'épaisseur de l'habiter, se mesurer au clair-obscur des situations perçues comme

\footnotetext{
${ }^{10}$ Le projet CECILE (Changement environnementaux côtiers : impact de l'élévation du niveau de la mer) est un projet de recherche partenariale public-privé soutenu par l'ANR et coordonné par Anny Cazenave (LEGOS-CNES) et Gonéri Le Cozannet (BRGM). Outre ces deux instituts, il a associé, entre 2010 et 2013, le Cerfacs, le Groupe d'étude de l'atmosphère météorologique du CNRS, le Créocéan, Météo France, le Shom et le laboratoire Littoral, environnement et sociétés de l'Université de La Rochelle.

${ }^{11}$ Programme du CNES «Espace, innovation et société » (2012-2016).
} 
inextricables par les acteurs «du terrain». Ces derniers sont saisis par un régime de défiance vis-à-vis des systèmes conçus pour faire face aux risques côtiers et, de proche en proche, par la critique généralisée des pouvoirs et des autorités qui, perdant toute légitimité, deviennent inaudibles, y compris lorsqu'ils portent de «bonnes causes »comme le climat...

\section{Les services climatiques: utopies technologiques ou ressources pour l'action}

Les travaux conduits sur les services climatiques, dernière thématique abordée, et plus particulièrement sur l'intégration de l'« information climat» dans des systèmes d'information géographique, invitent également à regarder avec circonspection les approches linéaires situant la science du côté de la production de connaissances et considérant l'action comme un enjeu de traduction opérationnelle, de communication et d'éducation des populations. Comme l'a rappelé Pascale Braconnot, l'enjeu est de donner une réalité tangible aux extrapolations des chercheurs en matière de changement climatique. De nombreuses activités industrielles sont sensibles aux facteurs météorologiques, mais la notion de changement climatique n'est pas toujours bien assimilée. Le défi consiste à intégrer dans les réflexions, et plus particulièrement dans l'appréhension de la vulnérabilité de l'entreprise, un fonctionnement inédit du climat. Les logiques du monde industriel, notamment les habitudes de confidentialité, s'accordent mal avec la complexité scientifique et ses incertitudes chroniques. Reste que les climatologues ont tendance à situer l'apport des sciences sociales surtout du côté de la communication ou du décryptage de la société. Dans le cadre du projet SECIF $^{12}$, des sociologues ont étudié la territorialisation de la problématique en Alsace. Leur enquête visait trois objectifs : identifier des attentes en matière de services climatiques, éclairer la recherche sur ces services et typifier les vulnérabilités et les postures des entreprises. Comme pour les paysans d'Afrique subsaharienne, la capacité des acteurs à modifier leurs pratiques en fonction des événements extérieurs qu'ils perçoivent ne débouche pas nécessairement sur une attente de services climatiques. Amandine Amat a développé dans sa thèse (2016) l'exemple d'un viticulteur ayant adapté ses méthodes de production après avoir pris conscience de l'évolution de la qualité de ses vins depuis dix ans. Pour lui, le problème majeur est de faire avec l'incertitude.

\footnotetext{
${ }^{12}$ Le projet SECIF (Vers des services climatiques pour les industriels français) est un projet soutenu par l'ANR entre 2010 et 2014.
}

La question de la réduction de l'incertitude est également essentielle pour les producteurs d'information météorologique. Les volumes importants de données générés par les dispositifs satellitaires ont constitué une ressource déterminante pour la prévision. Pour autant, comme Sylvain Lenfle le démontre dans le cadre d'un autre projet mené avec le $\mathrm{CNES}^{13}$, la disponibilité de ces données ne s'est pas traduite immédiatement dans l'efficacité des dispositifs opérationnels. L'assimilation de données satellitaires aux caractéristiques radicalement différentes s'est heurtée à des difficultés allant du rythme de production lié à la disponibilité des données jusqu'à la qualité même des modèles numériques de prévision du temps, fondés sur des données in situ et de sondage par ballon. Plusieurs années de coopération scientifique entre mathématiciens et météorologues ont été nécessaires pour identifier les problèmes et concevoir un autre mode d'assimilation, maintenant partagé par les agences météorologiques. S. Lenfle, spécialiste des processus d'innovation suggère une autre manière de concevoir les objets techniques, prenant en compte les méthodes d'assimilation des données, opérant le lien avec des écosystèmes d'usage et intégrant les compétences des utilisateurs.

\section{Émergence d'un nouveau paradigme?}

Les discussions du colloque montrent que la conception de l'adaptation comme une descente d'échelle du global jusqu'au local, voire jusqu'aux individus, néglige la dimension politique de l'habiter. Elle minimise les propriétés émergentes des milieux, y compris des milieux techniques qui contribuent à l'administration des territoires, à la gestion du quotidien comme à la maîtrise des événements «hors cadre», ainsi qu'à la production de l'information. La tentation est grande de mobiliser le seul régime de l'axiomatique autonome (Chateauraynaud et Debaz, 2017) pour produire des solutions clé en main là où les conditions inédites créées par le climat réclament le développement de nouvelles articulations entre connaissances et actions, entre raisonnements formels et expériences sensibles. La question est en réalité celle de la distribution des formes de vigilance. La solution technologique lie les acteurs à des réseaux industriels et des systèmes économiques, fonctionnant avec de fortes asymétries-même le web, qui était supposé incarner l'horizontalité d'un monde en réseau, est désormais sous le contrôle d'une poignée de méga-acteurs. La solution étatique, centralisée, est quant à elle génératrice de problèmes de bureaucratie et de conflits de compétences. En retour, une approche purement locale entraîne aussi des tensions, notamment des conflits d'intérêts, voire des

\footnotetext{
${ }^{13}$ Projet «La construction de la valeur des données spatiales », dans le cadre du programme du CNES «Espace, innovation et société» (2012-2016).
} 
phénomènes de corruption. La production des connaissances scientifiques engendrant des tensions épistémiques, entre certitudes et incertitudes, savoirs et ignorances, on ne peut y trouver refuge sans engendrer de nouvelles incomplétudes. Nul autre choix, pour conclure, que d'organiser une boucle de vigilance convoquant tour à tour toutes les échelles et toutes les instances pertinentes. Parmi celles-ci, les veilles citoyennes, les savoirs et les alternatives qu'elles produisent doivent jouer un rôle majeur, sans être réduits à l'activité de la «société civile organisée» via les grandes ONG. L'expérience de la citoyenneté doit être distribuée afin de permettre de saisir contraintes et potentialités, d'ouvrir et de fermer les processus critiques en se plaçant aux bonnes échelles d'action et de jugement.

Les besoins de connaissances et d'appui à l'action sont considérables. Reste que les programmes et dispositifs permettant aux chercheurs de travailler ensemble dans la durée sont rares : il y va donc aussi des politiques de la recherche et des conditions nécessaires pour reconstruire les problématiques à partir de réelles confrontations des approches, en visant à la fois la production de connaissances et l'engagement dans le monde social. Au-delà d'un simple plaidoyer pour des pratiques interdisciplinaires, ce sont donc bien de nouveaux cadres de saisie des objets environnementaux et de nouvelles manières de concevoir la climatologie qui sont en jeu : la fabrique des prises collectives sur le futur en dépend.

\section{Références}

Amat A., 2016. Le changement climatique de la simulation aux modes d'existence. Étude de trajectoires climatiques de villes et d'entreprises en Alsace. Thèse de doctorat en sociologie, Strasbourg, Université de Strasbourg.

Aykut S., Dahan A., 2015. Gouverner le climat? Vingt ans de négociations internationales, Paris, Presses de Sciences Po.

Blanc G., Demeulenaere E., Feuerhahn W. (Eds), 2017. Humanités environnementales : enquêtes et contre-enquêtes, Paris, Éditions de la Sorbonne.

Borie M., Hulme M., 2015. Framing global biodiversity: IPBES between mother earth and ecosystem services, Environmental Science \& Policy, 54, 2015, 487-496.
Charvolin F., Ollivier G., 2017. La biodiversité entre science et politique. La formation d'une institution internationale, Paris, Éditions Pétra.

Chateauraynaud F., 2016. Towards a new matrix of risks: learning from multi-scale controversies, in European Environment Agency, Report of the EEA Scientific Committee Seminar on emerging Systemic Risks, Copenhagen, 24 February 2016, 12-17.

Chateauraynaud F., Debaz J., 2017. Aux bords de l'irréversible. Sociologie pragmatique des transformations, Paris, Éditions Pétra.

Cometti G., 2015. Lorsque le brouillard a cessé de nous écouter. Changement climatique et migrations chez les Q'eros des Andes péruviennes, Berne, Peter Lang.

Crate S.A., Nuttall M., 2016 [1 ${ }^{\text {re }}$ éd. : 2009]. Anthropology and climate change. From actions to transformations, Routledge.

Daston L., Galison P., 2007. Objectivity, New York, Zone Books.

Edwards P., 2010. A vast machine. Computer models, climate data, and the politics of global warming, Cambridge [MA], The MIT Press.

Hulme M., 2013. Reducing the future to climate: a story of climate determinism and reductionism (abridged, with commentary), in Robin L., Sörlin S., Warde P. (Eds), The future of nature. Documents of global change, New Haven [CT], Yale University Press, 506-525.

Ingold T., 2011. Being alive. Essays on movement, knowledge and description, Oxon/New York, Routledge.

Joussaume S., Pacteau C., Péan C., Vautard R. (Eds), 2016. Approches interdisciplinaires du changement climatique. L'expérience du GIS Climat-Environnement-Société (2007-2016). Rapport, GIS Climat-EnvironnementSociété, http://www.gisclimat.fr/sites/default/files/Synth\% c3\%a8se\%20GIS_BD.pdf.

Le Treut H. (Ed.), 2013. Les impacts du changement climatique en Aquitaine: un état des lieux scientifique, Pessac, Presses universitaires de Bordeaux/LGPA Éditions.

Treyer S., Aubertin C., 2016. Les Conventions climat et biodiversité: une nouvelle géopolitique des rapports de force, Natures Sciences Sociétés, 24, 4, 317-318.

Tsing A.L., 2015. The mushroom at the end of the world. On the possibility of life in the capitalist ruins, Princeton, Princeton University Press. Trad. fr. : Le champignon de la fin du monde. Sur les possibilités de vivre sur les ruines du capitalisme, Paris, La Découverte, 2017.

Citation de l'article : Chateauraynaud F., Dubois C., 2019. Et si la climatologie devenait une science sociale comme les autres ? À propos du colloque « Entre connaissance et action : regards croisés sur les enjeux climatiques et environnementaux ». Nat. Sci. Soc. 27, 1, 63-72. 